\title{
Detection and Use of Load and Gage Output Repeats of Wind Tunnel Strain-Gage Balance Data
}

\author{
N. Ulbrich ${ }^{\dagger}$ \\ Jacobs Technology Inc., Moffett Field, California 94035
}

Criteria are discussed that may be used for the detection of load and gage output repeats of wind tunnel strain-gage balance data. First, empirical thresholds are introduced that help determine if the loads or electrical outputs of a pair of balance calibration or check load data points match. A threshold of 0.01 percent of the load capacity is suggested for the identification of matching loads. Similarly, a threshold of $0.1 \mathrm{microV} / \mathrm{V}$ is recommended for the identification of matching electrical outputs. Two examples for the use of load and output repeats are discussed to illustrate benefits of the implementation of a repeat point detection algorithm in a balance data analysis software package. The first example uses the suggested load threshold to identify repeat data points that may be used to compute pure errors of the balance loads. This type of analysis may reveal hidden data quality issues that could potentially be avoided by making calibration process improvements. The second example uses the electrical output threshold for the identification of balance fouling. Data from the calibration of a six-component force balance is used to illustrate the calculation of the pure error of the balance loads.

\section{Nomenclature}

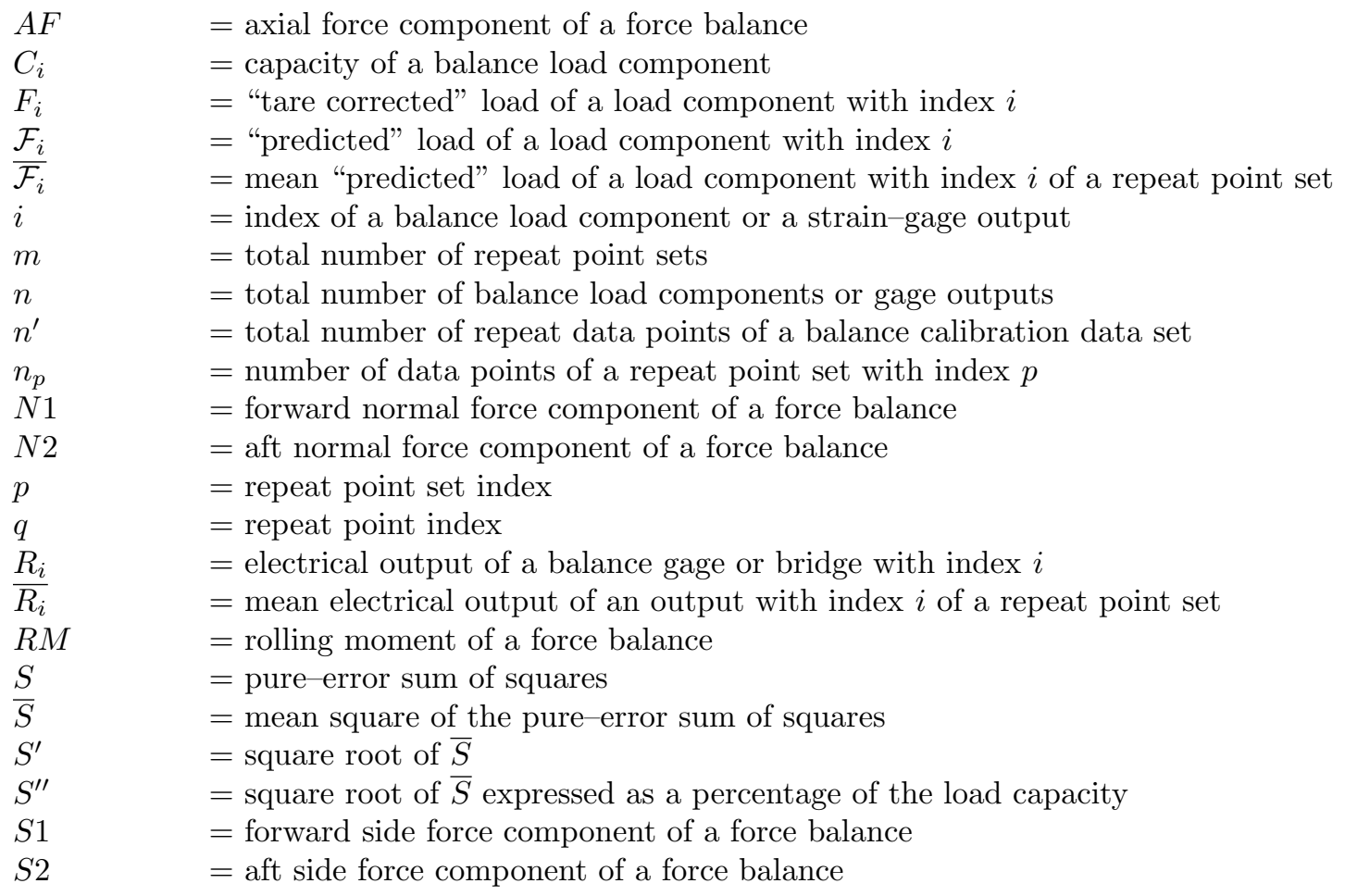

$\dagger$ Aerodynamicist, Jacobs Technology Inc. 


$$
\begin{aligned}
& \delta F_{i} \quad=\text { threshold used for the identification of repeat loads } \\
& \delta R_{i} \quad=\text { threshold used for the identification of repeat outputs } \\
& \Delta S 1, \Delta S 2 \quad=\text { calibration or repeat load residuals of the side force components of a force balance } \\
& \eta \quad=\text { data point index } \\
& \xi=\text { data point index }
\end{aligned}
$$

\section{Introduction}

A systematic analysis and evaluation of sets of repeat data points of both wind tunnel strain-gage balance calibration and check load data can provide useful insight into the overall quality of the collected experimental data. These repeat data points may be used, for example, to assess how well a specific characteristic of the balance can objectively be reproduced during calibration or check loading of the balance. Similarly, it is possible to use elements of a repeat point detection process for the automatic identification of balance fouling, i.e., for the detection of a situation when the balance calibration body comes into direct contact with the non-metric part of the balance. Therefore, a repeat point detection process and two related practical applications of the process were recently implemented in a wind tunnel strain-gage balance data analysis software package. Basic elements and ideas of the detection process and two examples of its application are discussed in the paper.

Empirical criteria are presented in the next section of the paper that may be used for the detection of load and output repeats. Then, the use of these criteria is demonstrated by describing the implementation of a pure error analysis algorithm that uses (i) the "tare corrected" loads for the identification of repeat points and (ii) the "predicted" loads of the repeats for the calculation of the pure error. This pure error analysis algorithm assumes that the Iterative Method is used for the balance data analysis (see Ref. [1], pp. 7-19, for specific details about the Iterative Method). Afterwards, the use of repeat point detection criteria for the automatic identification of balance "fouling" is discussed. Finally, data from the calibration of a six-component force balance is processed to illustrate some typical results of a pure error analysis of sets of repeat loads that were identified using the proposed repeat load threshold.

\section{Repeat Load and Output Detection}

In principle, an analysis of sets of repeat data points of a wind tunnel strain-gage balance data set requires empirical criteria that help determine if the loads or electrical outputs of a pair of data points match. These criteria may compare, for example, the absolute value of the difference between the loads or outputs of a data point pair with an empirical threshold. The loads or outputs of the data point pair are assumed to "match" if the difference between the loads or outputs is below the threshold. Ideally, empirical thresholds for the evaluation of the loads or outputs of the data point pairs should be defined as dimensionless quantities so that they can easily be applied to balances of different design and size.

First, the threshold definition for the detection of matching loads is discussed. It is assumed that all loads of a balance calibration data set are "tare corrected." This characteristic implies that loads associated with the weight of (i) the calibration equipment and (ii) the metric part of the balance were added to the raw "applied" loads. Then, it is possible to identify matching loads of the balance calibration data that belong to different load series as all "tare corrected" loads are referenced to the common absolute load datum of zero load. The author recommends the following empirical threshold for the detection of matching loads ...

THRESHOLD DEFINITION FOR REPEAT LOADS

$$
\begin{gathered}
\left|F_{i}(\eta)-F_{i}(\xi)\right| \leq \delta F_{i} \\
\text { where } \\
\delta F_{i}=0.0001 \times C_{i} \equiv 0.01 \% \text { of capacity }
\end{gathered}
$$


where $F_{i}$ is the "tare corrected" load value of the load component with index $i, \eta$ and $\xi$ are the indices of the two data points that are being compared, $\delta F_{i}$ is the empirical threshold that is used for the detection of matching loads, and $C_{i}$ is the capacity of $F_{i}$. The suggested load threshold, i.e., $0.01 \%$ of capacity, was intentionally chosen to be a very small value so that the detection of matching pairs of "tare corrected" loads would be very reliable even if data points are compared that belong to different load series.

Data points with matching electrical outputs can also be detected by using a threshold-based criterion. It is simply assumed that the outputs of the balance are reported as absolute voltages or as output differences relative to the natural zeros of the balance (natural zero $\equiv$ electrical output of a gage at zero absolute load). Then, the following empirical threshold may be used to identify matching outputs ...

\section{THRESHOLD DEFINITION FOR REPEAT OUTPUTS}

$$
\begin{gathered}
\left|R_{i}(\eta)-R_{i}(\xi)\right| \leq \delta R_{i} \\
\text { where } \\
\delta R_{i}=0.1 \mu \mathrm{V} / \mathrm{V}
\end{gathered}
$$

where $R_{i}$ is the electrical output of a gage (bridge) with index $i, \eta$ and $\xi$ are the indices of the two data points that are being compared, and $\delta R_{i}$ is the empirical threshold for the detection of matching outputs. Again, the recommended output threshold, i.e., $0.1 \mu \mathrm{V} / \mathrm{V}$, was intentionally chosen to be a very small value so that the detection of matching pairs of electrical outputs would be very reliable.

Practical applications of the two thresholds are discussed in the following sections of the paper. The first application looks at the calculation of the pure error of the balance loads assuming that the Iterative Method is used for both balance data analysis and load prediction. The second application illustrates how the threshold for matching outputs could be used to detect balance fouling.

\section{Pure Error Analysis of Balance Loads}

In general, a pure error analysis of experimental data is an important tool that evaluates the variation of the responses of repeat data points (see, e.g., Ref. [2], pp.145-149, for a description of elements of a pure error analysis). Results of this analysis may help identify data acquisition process imperfections or data quality issues as pure error analysis results are, by design, independent of the regression model that is used to fit the data.

In principle, a pure error analysis can be applied to wind tunnel strain-gage balance calibration data as long as a sufficient number of repeat points exist. However, the reader must not forget that the "tare corrected" calibration loads are the independent variables and the "measured" outputs of the gages are the dependent (or "observed") variables of the balance calibration process. Therefore, in theory, the electrical outputs should be used to compute the pure-error sum of squares of the balance calibration data set as the outputs are the "responses" of the experimental data. The resulting equation for the calculation of the pure-error sum of squares of an output with index $i$ has the following form (see Ref. [2], Eq. (4.21)) ...

$$
\begin{gathered}
S\left(R_{i}\right)=\sum_{p=1}^{m} \sum_{q=1}^{n_{p}}\left[R_{i}(p, q)-\overline{R_{i}}(p)\right]^{2} \\
\overline{R_{i}}(p)=\frac{1}{n_{p}} \cdot \sum_{q=1}^{n_{p}} R_{i}(p, q)
\end{gathered}
$$

where $R_{i}$ is an electrical output of a repeat point set with index $p, \overline{R_{i}}$ is the mean output of a repeat point set with index $p, m$ is the total number of repeat data point sets of the given data, $q$ is an index of a repeat point, and $n_{p}$ is the total number of repeats of the set of repeat points with index $p$. 
The required use of the dependent variables of the balance calibration data, i.e., of the "observed" electrical outputs, for the assessment of the pure error of a balance calibration data set has a disadvantage: balance users may have difficulties interpreting results of the pure error analysis because they often use the loads instead of the measured electrical outputs for the description of the overall data quality. Fortunately, it is possible to simply "interpret" the "predicted" loads resulting from the application of the regression model of the balance calibration data as "observed" loads. This interpretation is reasonable because (i) the loads are computed by using the "observed" electrical outputs as input, (ii) all loads of a repeat point set have approximately the same regression model dependent error, and, (iii) the calculation of the difference between an "observed" repeat load and the related mean observation removes most of the regression model dependent errors from the analysis. Then, after replacing the "observed" outputs with the "observed" loads in Eqs. (3a) and (3b), the corresponding equation for the calculation of the pure-error sum of squares of an "observed" load with index $i$ has the following form ...

$$
\begin{gathered}
S\left(\mathcal{F}_{i}\right) \approx \sum_{p=1}^{m} \sum_{q=1}^{n_{p}}\left[\mathcal{F}_{i}(p, q)-\overline{\mathcal{F}_{i}}(p)\right]^{2} \\
\overline{\mathcal{F}_{i}}(p)=\frac{1}{n_{p}} \cdot \sum_{q=1}^{n_{p}} \mathcal{F}_{i}(p, q)
\end{gathered}
$$

where $\mathcal{F}_{i}$ is a "predicted" load of a repeat point set with index $p, \overline{\mathcal{F}_{i}}$ is the mean "predicted" load of a repeat point set with index $p, m$ is the total number of repeat point sets, $q$ is an index of a repeat point, and $n_{p}$ is the total number of repeats of the set of repeat points with index $p$. The "observed" loads $\mathcal{F}_{i}$ are "predicted" by using, for example, the Iterative Method (see Ref. [1], pp. 7-19). This approach first fits the outputs as a function of the "tare corrected" calibration loads. Afterwards, a load iteration scheme is constructed from the regression models that may be used to "predict" loads from measured gage outputs.

It is useful to introduce the average of the square of the pure errors of the load component with index $i$. This quantity is also called the "mean sum square" of the pure error. It is defined by the following expression (taken from Ref. [2], Eq. (4.24)) ...

$$
\begin{aligned}
\bar{S}\left(\mathcal{F}_{i}\right) & =\frac{S\left(\mathcal{F}_{i}\right)}{n^{\prime}-m} \\
n^{\prime} & =\sum_{p=1}^{m} n_{p}
\end{aligned}
$$

where $m$ equals the total number of repeat point data sets. The definition of the "mean sum square" of the pure error, i.e., Eq. ( $5 a)$, has the disadvantage that it is expressed in terms of the square of the load units. Consequently, it is more difficult to interpret in a practical context. However, an analyst could simply use the square root of the right-hand side of Eq. $(5 a)$ as an alternate metric for the evaluation of the pure error of a set of repeat loads. This metric has, by design, a unit that matches the unit of the load component being evaluated. Then, Eq. ( $5 a$ ) takes on the following form ...

$$
S^{\prime}\left(\mathcal{F}_{i}\right)=\sqrt{\frac{S\left(\mathcal{F}_{i}\right)}{n^{\prime}-m}}
$$

where $n^{\prime}$ is defined in Eq. (5b) above. Finally, it is also useful to express the square root of the mean sum square of the pure error estimate as a percentage of the capacity of the load component in order to make the result less dependent on the size of the balance. Then, we get ...

$$
S^{\prime \prime}\left(\mathcal{F}_{i}\right)=\sqrt{\frac{S\left(\mathcal{F}_{i}\right)}{n^{\prime}-m}} \times \frac{100 \%}{C_{i}}
$$

It remains to identify the $m$ repeat point sets that are contained in the balance calibration data so that the sum of squares of the pure error of the load can be computed. The author suggests to use the "tare corrected" load set of this purpose as (i) all loads of this set have the common load datum of zero absolute 
load and (ii) it is used to fit the "observed" gage outputs. Then, repeat loads contained in different load series can easily be identified as the "tare corrected" loads are all referenced to the common load datum of zero absolute load. "Tare corrected" loads of a pair of data points are simply compared by using the load threshold definition that is given in Eqs. $(1 a)$ and $(1 b)$. Consequently, the following condition may be used to identify data points of a repeat point set that are needed for the pure error calculation:

\section{CONDITIONS FOR A REPEAT DATA POINT}

Two calibration data points $\eta$ and $\xi$ of an $n$-component balance are assumed to be "repeats" if the absolute value of the difference between the "tare corrected" loads of the two data points is less than (or equal to) $0.01 \%$ of capacity for all $n$ components.

$$
\begin{aligned}
& \left|F_{1}(\eta)-F_{1}(\xi)\right| \leq 0.0001 \times C_{1} \\
& \underbrace{\left|F_{n}(\eta)-F_{n}(\xi)\right|}_{\text {tare corrected loads }} \leq \underbrace{0.0001}_{\text {Eq. }(1 b)} \times C_{n}
\end{aligned}
$$

Again, it is important to clearly distinguish the "tare corrected" loads used exclusively for the identification of repeats in the balance calibration data set from the "predicted" loads that are used as input for the pure error analysis. A "tare corrected" load is identified by using the symbol $F_{i}$ (see Eqs. $(1 a),(1 b)$ ). It is the independent variable of the least squares fit of the gage outputs that the Iterative Method performs. It is also used to identify repeat points in the calibration data. A "predicted" load, on the other hand, is identified by using the symbol $\mathcal{F}_{i}$ (see Eq. $(4 a),(4 b)$ ). It is the result of processing the measured outputs by using the load iteration process that results from the regression analysis of the balance calibration data (see also Ref. [1]). It is used as an input for the pure error analysis.

The above condition for the identification of repeats has to be applied by going through the entire balance calibration data set on a point-by-point basis. A sample data point is picked, for example, assuming that matching data points may exist. Then, "tare corrected" loads of the remaining data points are compared with the loads of the sample data point by using the above conditions for the identification of a repeat. A data point is assumed to belong to the repeat point set of the sample data point if it fulfills the conditions.

Another important use of the ability to detect strain-gage balance data repeats is the identification of balance fouling. This application is discussed in the next section of the paper.

\section{Detection of Balance Fouling}

The reliable use of a strain-gage balance requires that two conditions are always fulfilled when loads act on the balance. Condition 1: The balance flexures are allowed to change shape over the entire load range of the balance without coming into surface contact with each other. Condition 2: The load path from the metric to the non-metric part of the balance does not change. Then, the first derivative of each gage output with respect to each load component will be a continuous function within the use envelope of the balance and, consequently, both accurate and robust regression models can be developed from the balance calibration data for the load prediction (this conclusion also applies to balances with "bi-directional" outputs as long as the region near zero load is excluded from the use envelope).

In theory, it is possible that Condition 1 gets violated if, for example, a balance experiences large combined loadings such that pairs of elastically deformed balance flexures come into surface contact with each other. This situation may be described as "internal" fouling of the balance. Condition 2 may get violated if the "transition zone" between metric and non-metric part of a highly loaded balance experiences large elastic deformations such that the non-metric part comes into direct contact with the calibration body. This situation may be better understood after reviewing different loading scenarios that may occur during balance calibration. Figure 1a below shows, for example, a moderately loaded balance during its calibration

American Institute of Aeronautics and Astronautics 
(the "transition zone" is marked as the yellow region between metric and non-metric part).

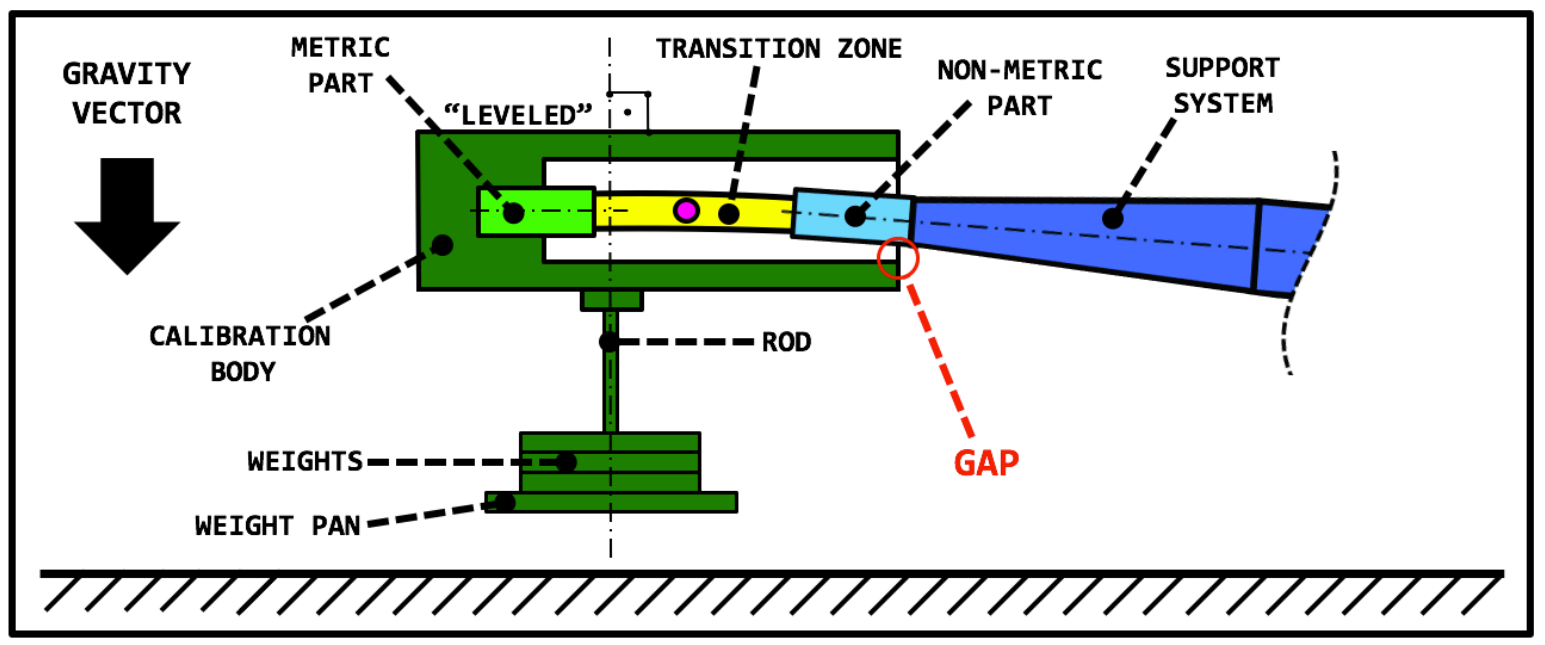

Fig. 1a Moderately loaded and leveled balance during calibration.

In this case, the balance will behave as intended as a "physical" gap exist between the calibration body and the non-metric part of the balance. Consequently, the load path from calibration body to non-metric part is well defined as the calibration body and the attached gravity weights are exclusively "supported" by flexures that connect the metric with the non-metric part (see also Fig. 1b below).

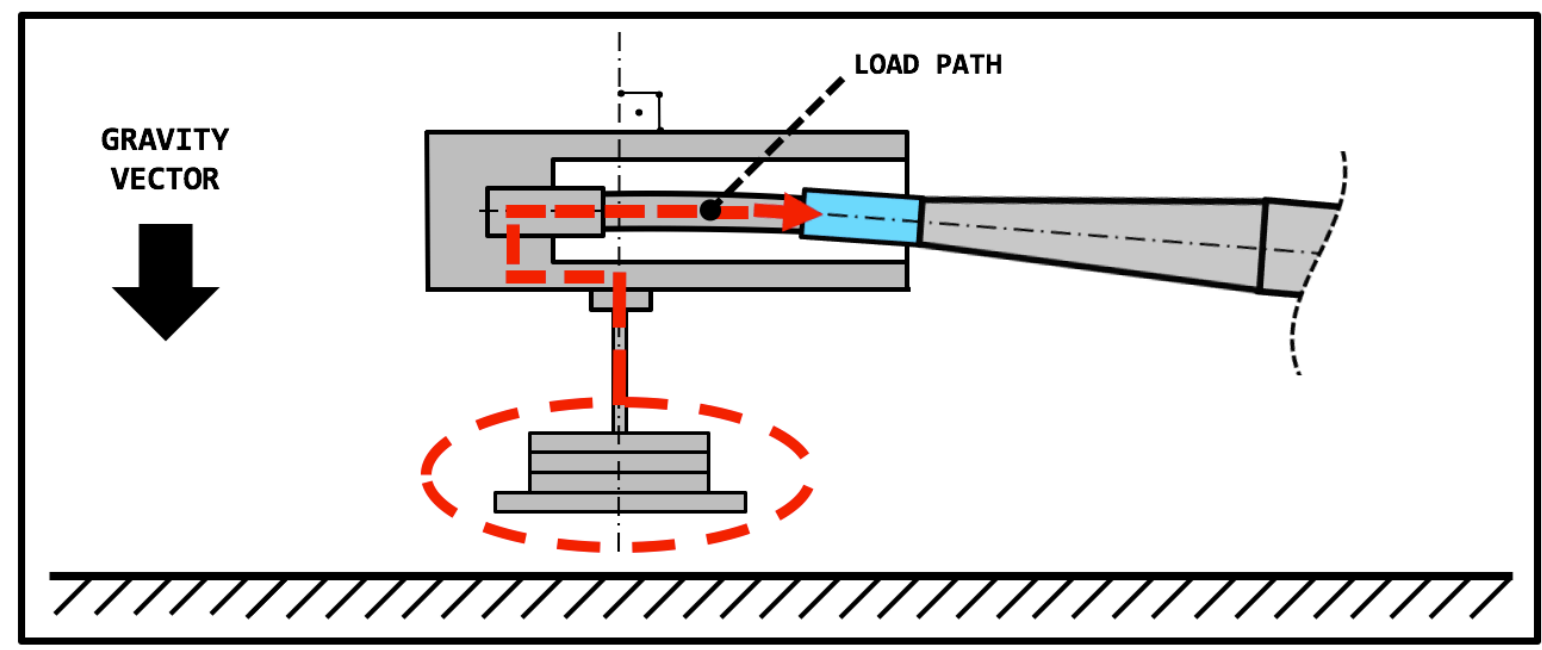

Fig. 1b Single load path of a moderately loaded balance.

Sometimes, however, a balance can experience very large loads such that the displaced calibration body comes into unintended "physical" contact with the non-metric part of the balance or the balance support system. This situation may be caused by a balance with a "transitional zone" that does not have enough stiffness as far as its elastic response to large loads is concerned (compare Fig. 1a with Fig. 2a below). 


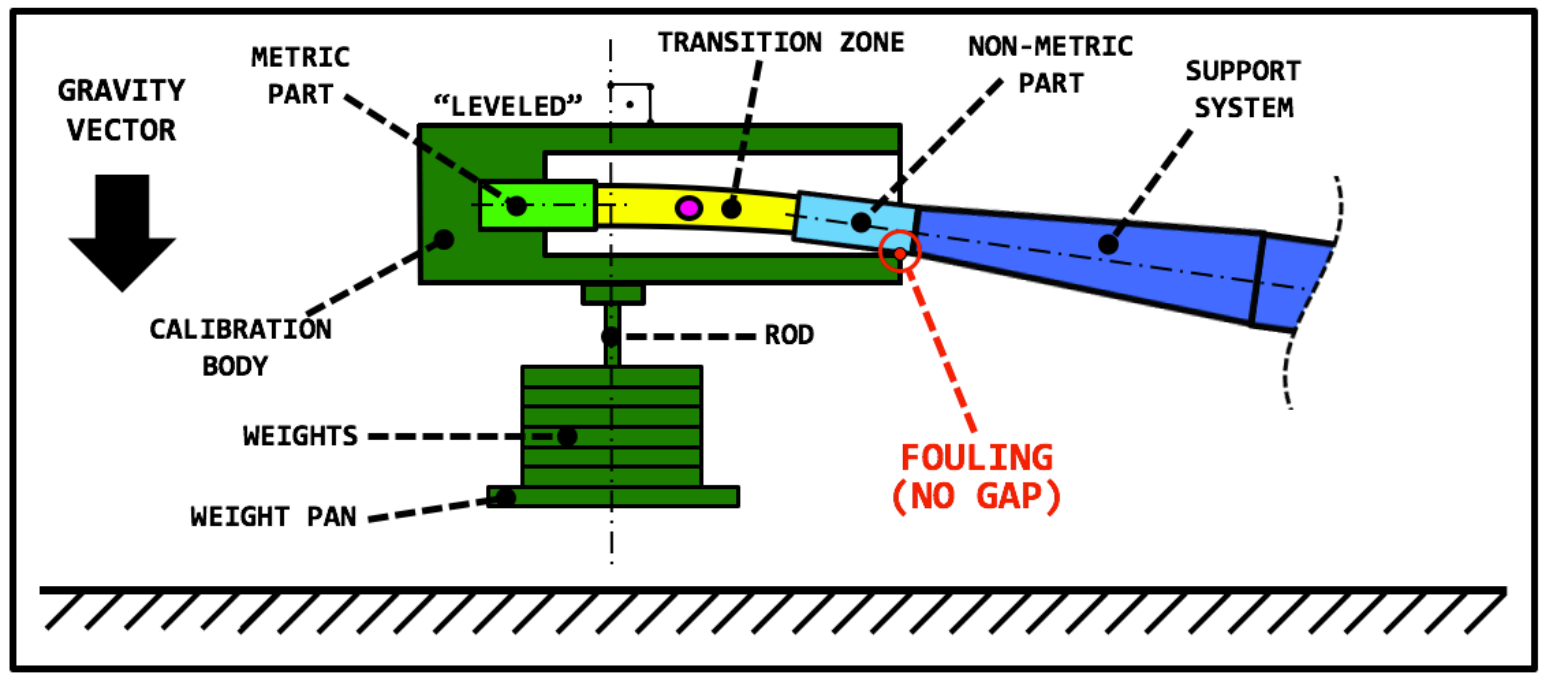

Fig. 2a Highly loaded, leveled, and externally "fouled" balance during calibration.

Then, Condition 2 above gets violated because the load path from the calibration body to the nonmetric part changed when compared with the unloaded state of the balance. Now, the calibration body and the attached gravity weights are "supported" by both (i) the flexures that connect the metric with the non-metric part and (ii) the contact point between the calibration body and the non-metric part (compare Fig. 1b with Fig. 2b below). This situation may be described as "external" fouling of the balance.

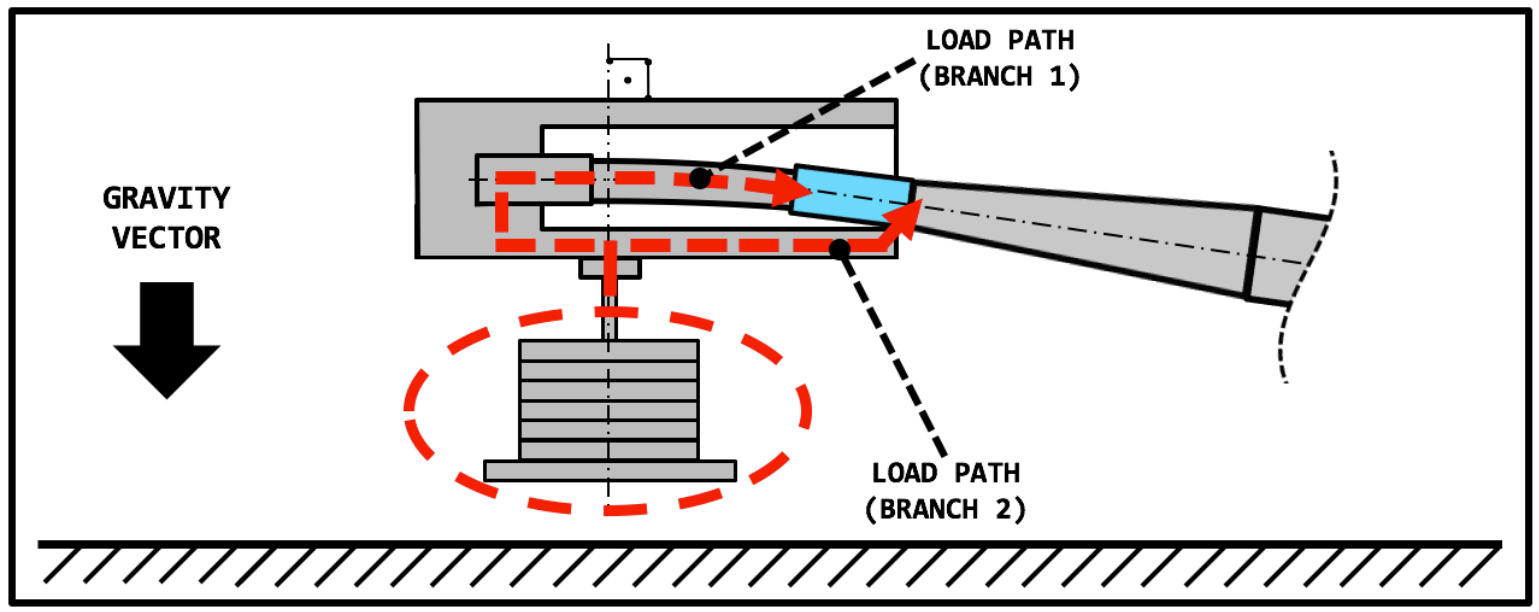

Fig. 2b Multiple load paths of a highly loaded and externally "fouled" balance.

Both "internal" and "external" fouling of the balance must be detected and avoided as those situations make balance load predictions unreliable. Often, an analyst plots all outputs of a balance versus a single load component in order to "visually" detect balance fouling. This detection approach works best if the given balance was calibrated using the so-called One-Factor-At-A-Time (OFAT) method (this method only changes a single load component within each load series while keeping all other load components unchanged).

It must not be forgotten that the successful "visual" detection of "fouling" also depends on the skill of an analyst to recognize certain patterns in plots. Alternatively, the proposed threshold for the detection of output repeats, i.e., Eq. $(2 a)$ and $(2 b)$, may be used to construct a detection algorithm that automatically screens balance data for "internal" and/or "external" fouling. Point-to-point load changes may simply be compared with related output changes in order to identify balance fouling. For example, the following sets of empirical conditions may be used to detect balance fouling: 


\section{CONDITIONS FOR BALANCE FOULING}

The calibration body is suspected to be in unwanted contact with the non-metric part of the balance if two sets of conditions are met: Set $1 \Longrightarrow$ the absolute value of the load difference of two consecutive data points with indices $\eta$ and $\eta+1$ exceeds $5 \%$ of capacity for at least one load with index $\lambda$ assuming that the load is loaded to at least $50 \%$ of capacity; Set $2 \Longrightarrow$ the absolute value of the output difference of at least one gage/bridge with index $\mu$ is less or equal $0.1 \mu V / V$ assuming that the same gage/bridge has an output range of greater or equal $20.0 \mu V / V$ within the related load series.

The empirical conditions above and associated thresholds were developed after analyzing and processing a wide range of balance calibration data sets. Again, similar to the "visual" balance fouling detection approach, the above criteria work best if the given balance was calibrated using the OFAT approach. - The calculation of the pure error of a balance calibration data set is used in the next section of the paper to demonstrate a practical application of the repeat point detection algorithm.

\section{Discussion of Example}

Calibration data of a strain-gage balance was selected to illustrate the calculation of the pure error of the load components. The chosen balance is NASA's MK40 six-component balance. The MK40 is a 2.5 inch diameter multi-piece force balance. Table 1 below lists capacities of the balance in force balance format.

Table 1: Load capacities of NASA's 2.5 inch MK40 six-component force balance.

\begin{tabular}{|c|c|c|c|c|c|}
\hline$N 1, \mathrm{lbs}$ & $N 2, \mathrm{lbs}$ & $S 1, \mathrm{lbs}$ & $S 2, \mathrm{lbs}$ & $R M$, in-lbs & $A F$, lbs \\
\hline 3,500 & 3,500 & 2,500 & 2,500 & 8,000 & 400 \\
\hline
\end{tabular}

The balance was calibrated in 2006 at the NASA Ames Balance Calibration Laboratory. The calibration data consisted of 164 loadings distributed across 16 load series. Figure 3 below summarizes basic properties of all "tare corrected" loadings and load combinations that were a part of the calibration data set.

\begin{tabular}{|c|c|c|c|c|c|c|c|}
\hline SERIES & POINTS & $\mathrm{N} 1$ & N2 & s1 & S2 & RM & AF \\
\hline 1 & 9 & $\begin{array}{c}+\mathrm{N} 1 \\
+1.3 \% \text { to }+64.1 \%\end{array}$ & $< \pm 2 \%$ & $< \pm 2 \%$ & $< \pm 2 \%$ & $< \pm 2 \%$ & $< \pm 2 \%$ \\
\hline 2 & 9 & $\begin{array}{c}-\mathrm{N} 1 \\
-64.1 \% \text { to }-1.2 \%\end{array}$ & $< \pm 2 \%$ & $< \pm 2 \%$ & $< \pm 2 \%$ & $< \pm 2 \%$ & $< \pm 2 \%$ \\
\hline 3 & 9 & $< \pm 2 \%$ & $\begin{array}{c}+\mathrm{N} 2 \\
+1.1 \% \text { to }+64.0 \%\end{array}$ & $< \pm 2 \%$ & $< \pm 2 \%$ & $< \pm 2 \%$ & $< \pm 2 \%$ \\
\hline 4 & 9 & $< \pm 2 \%$ & $\begin{array}{c}-\mathrm{N} 2 \\
-63.9 \% \text { to }-1.0 \%\end{array}$ & $< \pm 2 \%$ & $< \pm 2 \%$ & $< \pm 2 \%$ & $< \pm 2 \%$ \\
\hline 5 & 9 & $< \pm 2 \%$ & $< \pm 2 \%$ & $\begin{array}{c}+S 1 \\
+1.7 \% \text { to }+89.7 \%\end{array}$ & $< \pm 2 \%$ & $< \pm 2 \%$ & $< \pm 2 \%$ \\
\hline 6 & 9 & $< \pm 2 \%$ & $< \pm 2 \%$ & $\begin{array}{c}-\mathrm{S} 1 \\
-89.7 \% \text { to }-1.7 \%\end{array}$ & $< \pm 2 \%$ & $< \pm 2 \%$ & $< \pm 2 \%$ \\
\hline 7 & 9 & $< \pm 2 \%$ & $< \pm 2 \%$ & $< \pm 2 \%$ & $\begin{array}{c}+\mathrm{S} 2 \\
+1.6 \% \text { to }+89.6 \%\end{array}$ & $< \pm 2 \%$ & $< \pm 2 \%$ \\
\hline 8 & 9 & $< \pm 2 \%$ & $< \pm 2 \%$ & $< \pm 2 \%$ & $\begin{array}{c}\mathrm{S} 2 \\
-81.4 \% \text { to }-1.4 \%\end{array}$ & $< \pm 2 \%$ & $< \pm 2 \%$ \\
\hline 9 & 9 & $< \pm 2 \%$ & $< \pm 2 \%$ & $< \pm 2 \%$ & $< \pm 2 \%$ & $< \pm 2 \%$ & $\begin{array}{c}\text { AF } \\
+15.9 \% \text { to }+115.9 \%\end{array}$ \\
\hline 10 & 9 & $< \pm 2 \%$ & $< \pm 2 \%$ & $< \pm 2 \%$ & $< \pm 2 \%$ & $< \pm 2 \%$ & $\begin{array}{c}\text { AF } \\
-111.3 \% \text { to }-11.3 \%\end{array}$ \\
\hline 11 & 19 & $-3.6 \%$ to $-0.8 \%$ & $-3.4 \%$ to $-0.6 \%$ & $< \pm 2 \%$ & $< \pm 2 \%$ & $\begin{array}{c} \pm \mathrm{RM} \\
-50.0 \% \text { to }+50.0 \%\end{array}$ & $< \pm 2 \%$ \\
\hline 12 & 19 & $-6.5 \%$ to $-0.8 \%$ & $-6.3 \%$ to $-0.6 \%$ & $< \pm 2 \%$ & $< \pm 2 \%$ & $\begin{array}{c} \pm R M \\
-100.0 \% \text { to }+100.0 \%\end{array}$ & $< \pm 2 \%$ \\
\hline 13 & 9 & $\begin{array}{c}+\mathrm{N} 1 \\
+0.9 \% \text { to }+32.3 \%\end{array}$ & $\begin{array}{c}+\mathrm{N} 2 \\
+0.8 \% \text { to }+32.2 \%\end{array}$ & $< \pm 2 \%$ & $< \pm 2 \%$ & $< \pm 2 \%$ & $< \pm 2 \%$ \\
\hline 14 & 9 & $\begin{array}{c}-\mathrm{N} 1 \\
-32.3 \% \text { to }-0.9 \%\end{array}$ & $\begin{array}{c}-\mathrm{N} 2 \\
-32.1 \% \text { to }-0.7 \%\end{array}$ & $< \pm 2 \%$ & $< \pm 2 \%$ & $< \pm 2 \%$ & $< \pm 2 \%$ \\
\hline 15 & 9 & $< \pm 2 \%$ & $< \pm 2 \%$ & $\begin{array}{c}+S 1 \\
+1.3 \% \text { to }+45.3 \%\end{array}$ & $\begin{array}{c}+S 2 \\
+0.9 \% \text { to }+44.9 \%\end{array}$ & $< \pm 2 \%$ & $< \pm 2 \%$ \\
\hline 16 & 9 & $< \pm 2 \%$ & $< \pm 2 \%$ & $\begin{array}{c}-\mathrm{S} 1 \\
-45.5 \% \text { to }-1.5 \%\end{array}$ & $\begin{array}{c}-S 2 \\
-45.0 \% 10-1.0 \%\end{array}$ & $< \pm 2 \%$ & $< \pm 2 \%$ \\
\hline
\end{tabular}

Fig. 3 Tare corrected calibration load schedule of the MK40 balance. 
It was decided to analyze the data by using the Iterative Method that is described in Ref. [1]. Therefore, the balance outputs were first fitted as a function of the loads. Absolute value terms were included in the regression model of the outputs because the normal and side force gages of the balance have bi-directional characteristics. In addition, a tare load iteration was performed so that the influence of the weight of the calibration equipment on the measured gage outputs would be included in the analysis. Afterwards, a load iteration scheme was constructed from the coefficients of the fitted outputs so that loads could directly be predicted from the outputs.

The calibration data analysis was performed by using NASA's BALFIT regression analysis tool (see Ref. [3]). Figure 4 below shows, for example, the final load residuals of the forward side force component plotted versus the data point index for the 16 load series. Overall, the forward side force residuals of the ca-

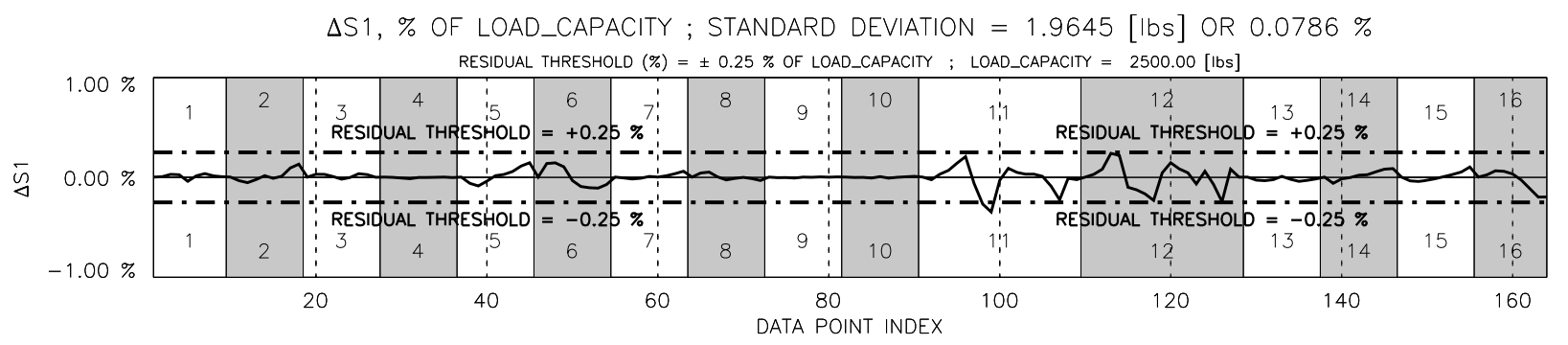

Fig. 4 Calibration load residuals of forward and aft side force of the MK40 balance.

libration data look good. They stay more or less within the traditionally used threshold of $0.25 \%$ of the load capacity.

Figure 5a below shows results of the pure-error analysis of the loads of the calibration data. A total number of 146 data points of all repeat point groups were found in the calibration data set $\left(\rightarrow n^{\prime}=146\right)$. They were distributed across 72 repeat data point sets $(\rightarrow m=72)$. Therefore, a total of 74 degrees of freedom were associated with the pure-error sum of squares of the loads $\left(\rightarrow n^{\prime}-m=74\right)$. The first row of Fig. 5a lists the "pure-error sum of squares" for each load component that was computed by using Eq. (4a). The second row lists the "mean square of the pure-error sum of squares" for each load component. This quantity was computed by using Eq. (5a). The third row lists the "square root of the mean square of the pure error sum of squares" in engineering units. This quantity was computed by using Eq. (6). Finally, the bottom row lists the "square root of the mean square of the pure error sum of squares" expressed as a percentage of the load capacity. This result was computed by using Eq. (7).

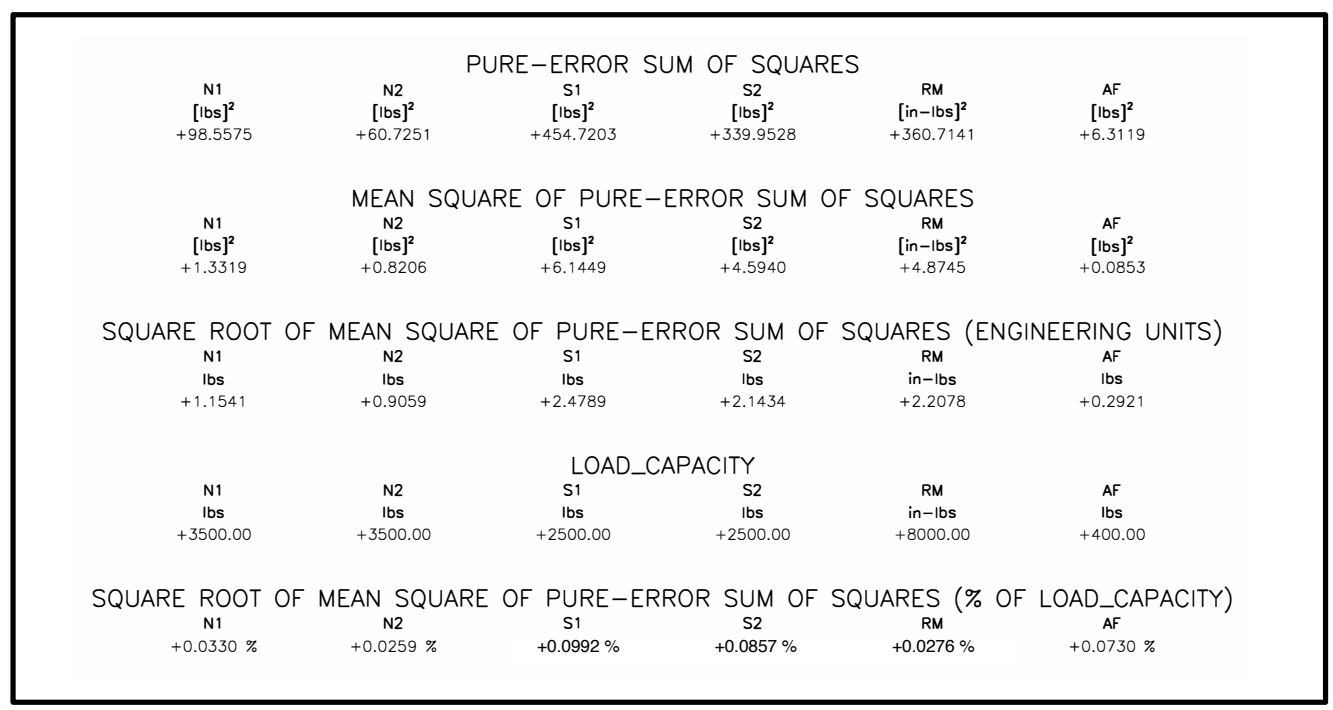

Fig. 5a Results of the pure error analysis of the MK40 balance calibration data.

American Institute of Aeronautics and Astronautics 
In principle, the "repeatability" of a load prediction can be quantified by the "square root of the mean square of the pure-error sum of squares" of the loads of repeats (see results listed on the bottom row of Fig. 5a). Numerical results shown in Fig. 5a indicate that the "repeatability" of the forward/aft side forces and the axial force are not as good as the "repeatability" of the other three load components as the corresponding numerical values are $0.0992 \%$ for $S 1,0.0857 \%$ for $S 2$, and $0.0730 \%$ for $A F$. Load predictions of these three components should be studied in more detail. For simplicity, the author decided to focus his investigations on the forward side force. Figure $5 \mathrm{~b}$ below shows the repeat load residuals of the forward side force of the calibration data set (repeat load residuals $\equiv$ load differences of the repeat points).

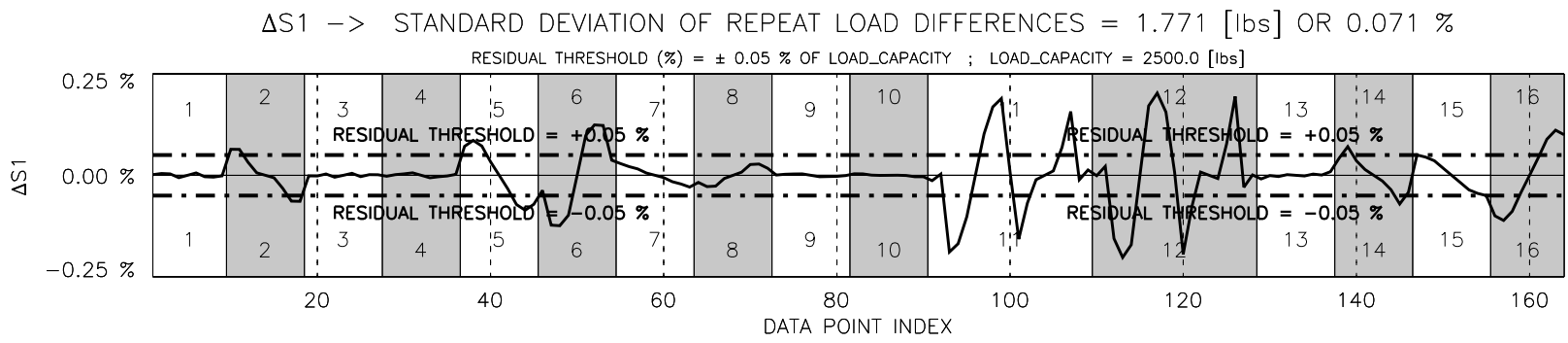

Fig. 5b Repeat load residuals of the MK40 balance plotted versus load series and data point index.

It can clearly be seen in Fig. 5b that the "repeatability" issues associated with the forward side force component originate in load series 11 and 12. In addition, it is known from the calibration load schedule shown in Fig. 3 that a rolling moment at constant normal force was applied during those two load series. Therefore, it appears that hidden calibration process imperfections negatively influenced the recording of repeats when the rolling moment was applied during the calibration of the MK40 balance.

\section{Summary and Conclusions}

Empirical thresholds were presented that may be used to automatically detect both load and gage output repeats in strain-gage balance calibration and check load data. The threshold for the detection of repeat loads is suggested to be $0.01 \%$ of the load capacity. The threshold for the detection of output repeats is recommended to be $0.1 \mu \mathrm{V} / \mathrm{V}$. Both thresholds are intentionally defined as dimensionless constants so that they can universally be applied to strain-gage balances of different sizes and capacities.

Two applications were discussed that require a reliable detection of matching loads or outputs of straingage balance data. The first application uses the threshold associated with the detection of repeat loads in order to identify sets of repeat points that are needed for the calculation of pure-error estimates of the balance loads. The second application uses the threshold associated with the detection of repeat outputs for the automatic identification of balance fouling. Data from the manual calibration of a force balance was used to show typical results of the pure-error analysis of the balance loads.

\section{Acknowledgements}

The author wants to thank Tom Volden of Jacobs Technology Inc. for his critical and constructive review of the final manuscript. The work reported in this paper was supported by the Wind Tunnel Division at NASA Ames Research Center under contract NNA16BD26C.

\section{References}

${ }^{1}$ AIAA/GTTC Internal Balance Technology Working Group, Recommended Practice: Calibration and Use of Internal Strain-Gage Balances with Application to Wind Tunnel Testing, AIAA R-091-2003, published by the American Institute of Aeronautics and Astronautics, Reston, Virginia, 2003.

${ }^{2}$ Montgomery, D. C., Peck, E. A., and Vining, G. G., Introduction to Linear Regression Analysis, 4th ed., John Wiley \& Sons, Inc., New York, 2006; pp. 145-149.

${ }^{3}$ BALFIT - Software Tool for the Regression Analysis of Multivariate Data - Version 3.01.21, distributed by the Software Release Authority, Technology Partnerships Office, NASA Ames Research Center, Moffett Field, California, December 2014. 